\title{
Development of Bioelectrical impedance analyzer for Body composition analysis
}

\author{
Ms. Shruti R Joshi ${ }^{1}$, Prof. U R. Bagal ${ }^{2}$ \\ I'Biomedical Engineering, MGMCET/ Mumbai University, India) \\ 2'(Biomedical Engineering, MGMCET/ Mumbai University, India)
}

\begin{abstract}
The human cells has different electrical conductivities. Due to difference in conductivities of cell constituents we can determine the electrical changes occurred in every constituent and characterized the human body to determine its composition such as fat free mass (FFM), fat mass (FM), total body water (TBW), Body mass index (BMI) and many other. Bioelectrical Impedance analysis (BIA) may be used for estimating body composition. It can be measured by applying a small $1 \mathrm{~mA}, 50 \mathrm{KHz}$ current which is injected into the body through a pair of electrodes and the induced voltage is picked up using another pair of electrodes. The impedance offered by the body to the applied current and the phase angle between the injected current and induced voltage is measured. The advantages of bioelectrical impedance method are non-invasive measurement, portability, safety, ease to use, low cost and acceptable levels of reliability and accuracy in estimation of body composition. A battery operated low cost and compact bio-impedance meter is developed which can be used for estimation of body composition parameters. It was proposed to measure body impedance and phase angle from healthy subjects using four electrode systems. The impedance values are recorded for different female and male individuals.
\end{abstract}

Keywords: Bio impedance, Body composition, Impedance, Phase angle.

\section{Introduction}

Body composition is the amount of lean tissue compared to fat. Body composition analysis can be used for estimation of the muscle and fat loss or gain due to exercise, nutrition, or pharmaceutical prescription which changes the body composition parameters. It can be categorized as fat mass (FM), fat free mass (FFM) and total body water (TBW) [1]. Several methods are used for estimation of body composition, including laboratory methods such as densitometry, computed tomography, electrical conductivity, amount of body water radio isotope dilution, whole-body counting of potassium and neutron activation. These methods are $\mathrm{s}$ are expensive and are not suited for field studies. Other techniques developed for epidemiological surveys, such as anthropometry, skinfold thickness measures, and infrared interactance are less reliable predictors of body composition. Thus there is a need for a safe noninvasive technique that is rapid, convenient and provides reliable and sufficiently accurate estimates of human body composition outside the laboratory. Measurement of wholebody bioelectrical impedance is an approach that may meet this need.

Bioelectrical impedance analysis (BIA) is simple, non-invasive, low cost widely used method for estimating body composition. BIA is currently used in diverse settings, including private clinicians' offices, health clubs, and hospitals, and across a spectrum of ages, body weights, and disease states. It is based electrical conductivity of the various body tissues. It involves an injection of low level application of a constant low-level alternating current results in impedance to the spread of the current that is frequency dependent. The living organism contains intra- and extracellular fluids that behave as electrical conductors and cell membranes that act as electrical condensers and are regarded as imperfect reactive elements. At low frequencies $(1 \mathrm{KHz})$ the current mainly passes through the extracellular fluids, whereas at higher frequencies $(500-800 \mathrm{KHz})$, it penetrates the intra- and extracellular fluids. The electrical conductivity depends on the electrolytes and body fluids whereas the cell membrane offers capacitive reactance due to its dielectric nature. Bioelectrical impedance measurements have been related to biological function such as pulsatile blood flow, cardiac output and estimation of body composition in healthy and diseased individuals [2].The Bioelectrical Impedance analyzer system is developed for estimation of Body composition. The phase angle between injected current and induced voltage was measured from healthy subjects of different age groups.

\subsection{Review of Literature}

Electrical properties of tissues have been described since 1871. In 1959, Nyboer [3] reported that the resistance of biological conductor is same as non-biological conductor. Later on Schwan and Kay [4], [5] reported that reactance of the bio impedance is supported by capacitive effect of cell membrane, tissue interfaces, and structural features. The capacitive effect depends on the frequency of the injected current. Lukaski and Bolonchuk [6] reported that the phase angle is important for describing relationships between 
bioelectrical impedance and physiological variables [7]. Early studies of bioelectrical impedance focused on impedance measures in relation to the water and electrolyte content of the body and to physiological variables such as thyroid function, basal metabolic rate, estrogenic activity and blood flow in human and animal tissues. The use of bioelectrical impedance to estimate body composition is developed from more recent exploratory works in the areas of single-frequency and multiple-frequency impedance [8], [9].

Nyboer and Hoffer[10],[11] first used single-frequency measures of impedance at $50 \mathrm{kHz}$ to estimate TBW based upon a volumetric relationship of impedance and a conductor using the impedance index. Thomasset, Jenin et al. [12], [13], first used measures of impedance at more than a single frequency to describe the proportion of extracellular water in TBW. These investigators used a ratio of impedance at low and high frequencies to differentiate this extracellular total-body relationship. By the 1970s the foundations of BIA were established, including those that underpinned the relationship between the impedance and the body water content of the body. The first application of BIA occurred in 1981. William and Admiral, in the US Navy, initiated a study to assess the hydration status of soldiers in high altitude cold weather environments. A variety of single frequency BIA analyzers then became commercially available and by the 1990s the market included several multi-frequency analyzers. More recently, segmental BIA has been developed to overcome inconsistencies between resistance (R) and body mass of the trunk.

\section{Bioelectrical Impedance and Body composition}

Bioelectrical impedance analysis (BIA) is the study of the electrical properties of biological material and its change over time. Human blood circulation and breathing has been quantified with a real-time BIA instrument [14]. The most popular application of BIA is estimating human body composition and body fluid status. In BIA, a small alternating current less than or equal to $1 \mathrm{~mA}$ is injected into the body and by measuring the voltage drop across the current path. The bioelectrical parameters such as resistance, reactance, phase angle etc. can be are derived from the measurement of bioelectric impedance. This method can be used to measure blood flow, monitor heart functions and perform similar functions for medically related research and patient treatment. BIA gives medical practitioners the opportunity to make differential diagnosis. It also forms the basis of objective, long-term approaches to treatment. An increasingly broad spectrum of instruments and software for specific areas of application ensure precision and safety in diagnosis. Many equations are available to estimate TBW and FFM as a function of impedance, weight, height, gender and age. The BIA calculations of an individual's body fat may vary by as much as 10 percent of body weight because of differences in machines and methodologies used. Equations and their variables differ as does the choice of a reference method. There is a need for a consensus among experts on the appropriate conditions of use and appropriate applications of BIA.

\subsection{Clinical Significance}

It is valuable to measure the body composition of the malnourished patients and to monitor their response for refeeding. The use of direct measurement techniques for clinical monitoring is expensive. So bioelectrical impedance analysis can be used. Malnutrition significantly alters body composition. The effects of malnutrition on body composition are well documented. Energy malnutrition results in wasting of body fat and lean mass. Protein malnutrition results in proportionately greater wasting of lean than fat mass. Further, within lean mass, intracellular or body cell mass is depleted relative to extracellular mass. Within the extracellular mass there is a contraction of plasma volume. The clinical correlate of this process is the occurrence of edema. Within the body cell mass, protein, and intracellular potassium concentration are reduced. These changes cannot be determined with height and weight measurements. The Bioelectric impedance analysis as a convenient clinical technique for estimating body composition analysis [15].

The clinical uses of BIA frequently concern conditions where water distribution is unbalanced, such as during critical illness. The assumptions of impedance and TBW are invalid in these cases. Historically, the applications of BIA have been focused on human body composition. Although literature describes a wide variety of various disease or population specific applications, the migration of BIA technology from research to clinical applications has been slow. One possible cause may be the fact that the potential usefulness of BIA is so widespread and involves so many different medical specialties. [16]. 


\subsection{Principle of Bioelectrical Impedance Analysis}

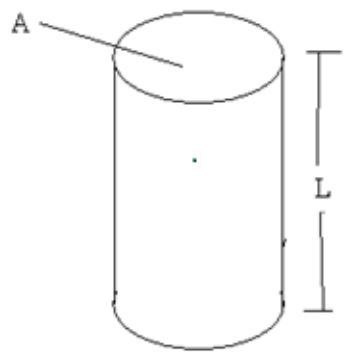

Figure 2.1 Cylinder of uniform resistivity

Consider a cylinder having cross sectional area $\mathrm{A}^{2}$ and length $\mathrm{L} \mathrm{m}$ of uniform resistivity as shown in Fig. 2.1, the impedance of the cylinder can be calculated as,

$$
Z=\frac{\rho L}{A} \text { (2.1) }
$$

Where,

$\mathrm{Z}=$ Impedance of biological and geometrical system in ohm, $\rho=$ Volume resistivity in ohm-cm.

$\mathrm{A}=$ Conductor cross sectional area in $\mathrm{cm}^{2}, \mathrm{~L}=$ Conductor length in $\mathrm{cm}$, Multiplying by $\mathrm{L}$ to numerator and denominator of R.H.S., we get

$$
\begin{aligned}
& Z=\rho \frac{L L}{A L} \\
& Z=\rho \frac{L^{2}}{V}
\end{aligned}
$$

Where, $\mathrm{V}=$ Volume of cylinder $=\mathrm{A} \times \mathrm{L}$

$$
V=\rho \frac{L^{2}}{Z}
$$

Thus from equation (2.2), for a uniform cylinder, conductor volume is directly proportional to the square of the length of the conductor and inversely proportional to the impedance of the conductor [16]. The hypothetical relationship between impedance and electrical volume was proposed by Nyober who demonstrated that electrically determined biological volume is inversely related to impedance of geometrical system [11].

$$
Z=R^{2}+X_{c}^{2}
$$

Where, $\mathrm{R}=$ Resistance of geometrical system, $\mathrm{Xc}=$ Reactance of geometrical system.

\subsection{Behavior of a Cell}

The conduction of an alternating electric current in the body is through its water content or, more correctly, the solution of electrolytes in the body. An alternating current is used for bioelectrical impedance analysis because it penetrates the body at low levels of voltage and age. In a complex electrical structure such as the human body, the part of the fluid volume measured by bioelectrical impedance is also a function of the current frequency. At low frequencies of less than about $10 \mathrm{kHz}$, the current travels primarily through extracellular fluids but as the frequency increases, the current starts to penetrate body tissues, and creating reactance as shown in Fig. 2.2 At high frequencies (above $100 \mathrm{kHz}$ ), the current is assumed to penetrate all conductive body tissues or all of the total body water in the conductor and supposedly overcomes the capacitive properties of the body, reducing reactance to zero. This implies that in a biological system the impedance at low frequency is related to the extracellular water (ECW) while the impedance at high frequencies is related to TBW [10]. Each cell is surrounded by a membrane that envelops intracellular fluids. At low frequencies $(<10 \mathrm{kHz})$, the cellular membrane has a high resistance and, therefore, the current travels through the extracellular fluids around the cells. At high frequencies, the capacitance of the cellular membrane short circuits the resistance of the membrane. 


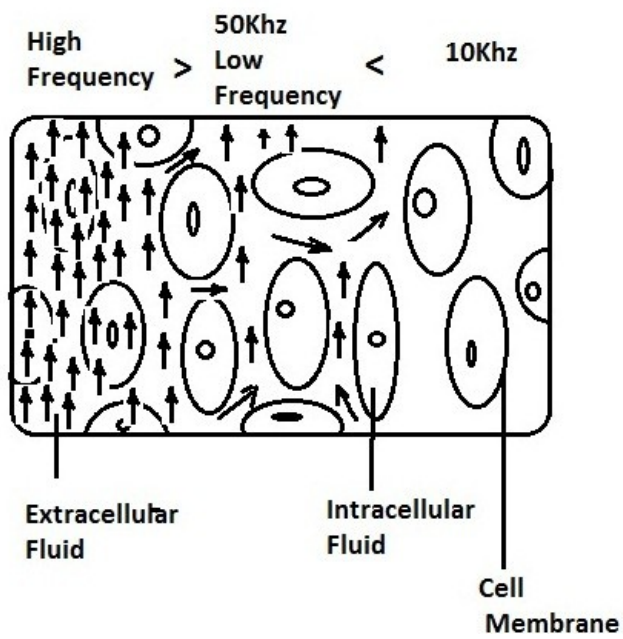

Figure 2.2 Flow of alternating current at different frequencies in body tissue.

\subsection{Bioelectrical Impedance of biological tissue}

The impedance of biological tissue comprises two components, the resistance and reactance (capacitive). Body fat, TBW and Extra-cellular water (ECW) offer resistance to electrical current while only cell membranes offer capacitive reactance. The cell membrane has a passive role to separate the extra and intracellular media and an active role to control the exchange of different chemical species. The passive part of the cell membrane is BLM. This film ( $\sim 7 \mathrm{~nm}$ thick) allows lipids and water molecules to pass through it but, in principle, it is completely closed for ions. Its intrinsic electrical conductance is very low and it can be considered as dielectric. Therefore the structure formed by extracellular medium, the BLM and the intracellular medium is a conductor- dielectric-conductor structure and it behaves as a capacitor $\left(\sim 1 \mu \mathrm{F} / \mathrm{cm}^{2}\right)$. Fig. 2.3 shows the bilayer lipid membrane and its electrical equivalent circuit. Thus cell membranes offer capacitive reactance. Since fat tissue cells are not surrounded by cell membranes, reactance is not affected by the quantity of body fat. In parallel with the BLM there are embedded proteins, transport organelles, ionic channels and ionic pumps. These structures are the basic elements of the membrane active role. The ionic channels are the porous structure that allows some ions to flow from the outside to inside of the cell or vice versa. These structures are selective to the ions and can be opened or closed by some electrical or chemical signals [16].

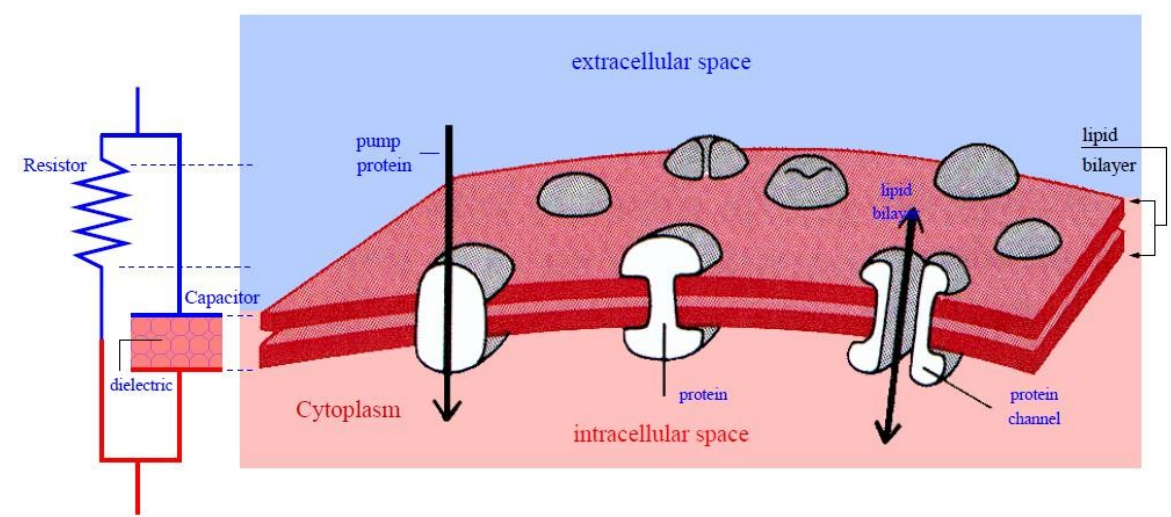

Figure 2.3 The plasma membrane of the cell and its electrical equivalent circuit [16].

\subsection{Measurement techniques for BIA}

In order to avoid tissue damage or electrode degradation, most electrode used for bioelectrical impedance measurements are made with noble metals (Pt or $\mathrm{Au}$ ) or stainless steel. In the interface of these electrodes with the tissue, no electronics exchange reaction exists and, as a consequence, the direct current is not able to flow through them from the metal to the tissue or vice versa. Hence these electrodes are called blocking electrodes and only the alternating current is able to flow through them. Its impedance is quite similar to a capacitance that depends on the electrode area inversely. In general in bio impedance technique a small alternating current of constant magnitude equal to $1 \mathrm{~mA}$ and high frequency is introduced into the body using a couple of electrodes called as current electrodes and the resulting voltage drop is measured with another couple of electrodes called as voltage electrodes. This method is known as four-electrode or tetrapolar method as shown in Fig. 2.4. It ideally minimizes the influence of the electrode-electrolyte interface impedance [4]. 


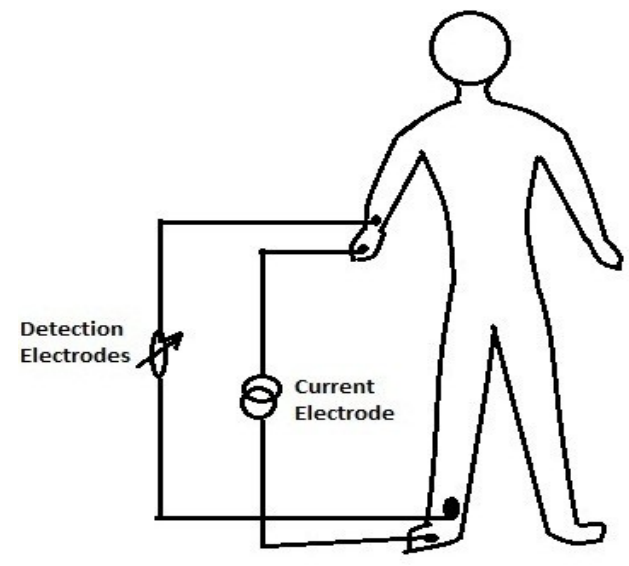

Figure 2.4 Placements of Electrodes.

\subsection{Body Composition}

A human body is composed of water, protein, bone and fat, in addition to a small amount of special components. The total of these elements constitutes the body weight. Quantitatively measuring the respective elements is called body composition analysis. The composition of human body creates natural interest. Scientists have been studying body composition since the beginning of the $20^{\text {th }}$ century but research has increased dramatically in the last 25 years. Body composition data can form the basis for a wide variety for therapeutic health and fitness prescriptions. In clinical applications body composition analysis along with nonpharmacologic nutrition and exercise prescriptions provide the foundation upon which further treatment is based. Only body composition analysis can determine how much muscle or fat is lost or gained as a result of any nutrition, exercise or pharmaceutical prescription. Human body mass may be conceptually divided into numerous fractional masses by assuming the different qualities of body tissues, water holding qualities and differential densities of various tissues. On the basis of these qualities, model may be conceived as a range from a single compartment to multi-compartment.

The one-compartment model (Figure 2.5-A), working as the most basic parameter, corresponds to measurement of body weight. Being the only compartment, it does not take into consideration factors of an individual's physique. In the two-compartment model (Fig.2.5-B) the body is divided into fat mass and fat free mass. Further the fat free mass is separated (Fig.2.5-C) into lean body mass (LBM) and bone mineral content (BMC). The three-compartment model (Fig.2.5-F) arises by subdividing the Fig. 2.5 Compartment models of body composition. One-compartment model (A); two-compartment model (B); three-compartment model (F); $\mathrm{BMC}=$ bone mineral content. Fat free mass into the body cell mass (BCM) and the extracellular mass (ECM). $\mathrm{BCM}$ is the functional mass of the body where work is done. All oxygen consumption, carbon dioxide production, glucose oxidation, protein synthesis and other metabolic work takes place within the body cell mass. ECM is the support mass and is metabolically inactive, consumes no oxygen, produces no carbon dioxide and performs no work. The ECM consists of extracellular fluids and solids, such as bone and cartilage, with its primary function that of support and transport. Measurements in the three-compartment model are possible with phase sensitive multi-frequency BIA, total body potassium, dilution methods.

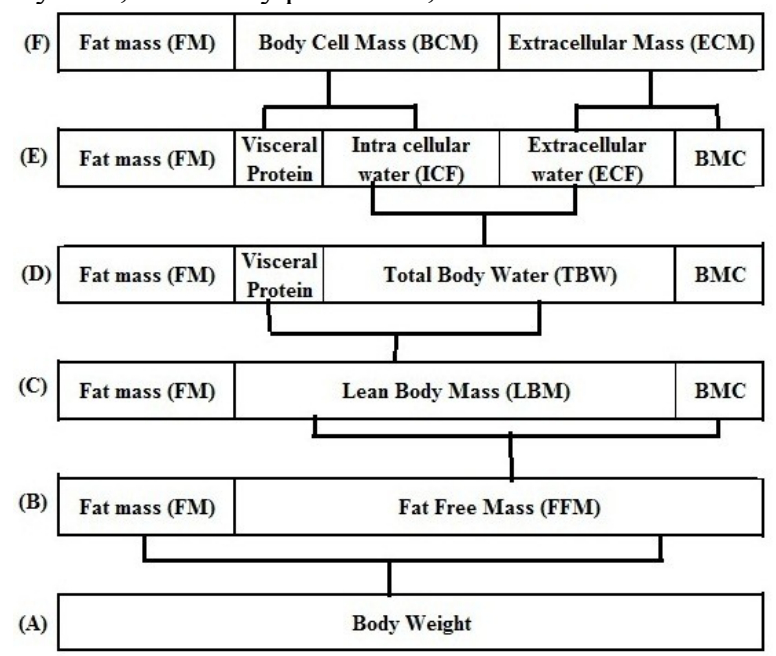

Figure 2.5 Compartment models of body composition. 


\subsection{Estimation of Body composition}

In BIA method human body is assumed to be a uniform cylinder, so in equation (2.3) we can replace impedance of cylinder $(\mathrm{Z})$ by Impedance of the body $\left(\mathrm{Z}_{\mathrm{body}}\right)$; length of cylinder $(\mathrm{L})$ by height of body $(\mathrm{h})$ and volume of the cylinder $(\mathrm{V})$ by volume of the conducting material in the body i.e. volume of the TBW. Thus by analogy, at high frequency (above $10 \mathrm{kHz}$ ) of alternating current, equation (2.3) becomes

$$
\text { VolumeOfTBW }=\rho \frac{h^{2}}{Z_{\text {body }}}
$$

The equation (2.5) is the base for the calculation of TBW. This relationship has been used by some investigator to predict conducting volume of total body water. $\mathrm{h}^{2} / \mathrm{Z}_{\text {body }}$ is also called as impedance quotient (or impedance index) and many studies have confirmed that the impedance quotient is highly correlated with TBW and therefore, assuming a constant hydration factor (73\%) for the FFM, FM can be estimated as

$$
\begin{aligned}
& \text { FatFreeMass }(F F M)=\frac{\text { TotalBodyWater }(T B W)}{0.73} \\
& \text { FatMass }(F M)=\text { Weight }-F F M \quad(2.7)
\end{aligned}
$$

The equations (2.5) to (2.7) give the body composition. When these equations are applied to the body, many assumptions are made including homogeneity, uniform current distribution and uniform cross sectional area. However, the geometrical relationship in the equation (2.5) is between the value of impedance and the volume of the TBW is not adequately explained in the literature. The uniformity is not a characteristic of the human body or of human populations. The specific resistivity, or $\rho$, in this volumetric equation (2.5) is an electrical property particular to the conducting medium and independent of its size or shape. For a homogeneous conductor, it is a constant physical property similar to specific gravity. Specific resistivity for the whole body is assumed to be a constant but each tissue has a characteristic specific resistivity [16].

\section{System Configuration and Instrumentation.}

Based on bio impedance principle and the single frequency measurement technique the aim is to develop a totally portable instrument, which computes body impedance and phase angle parameters which are required to estimate body composition and also have low cost and require less time and portability feature. System block and circuit design is explained in details in this heading.

\subsection{System configuration}

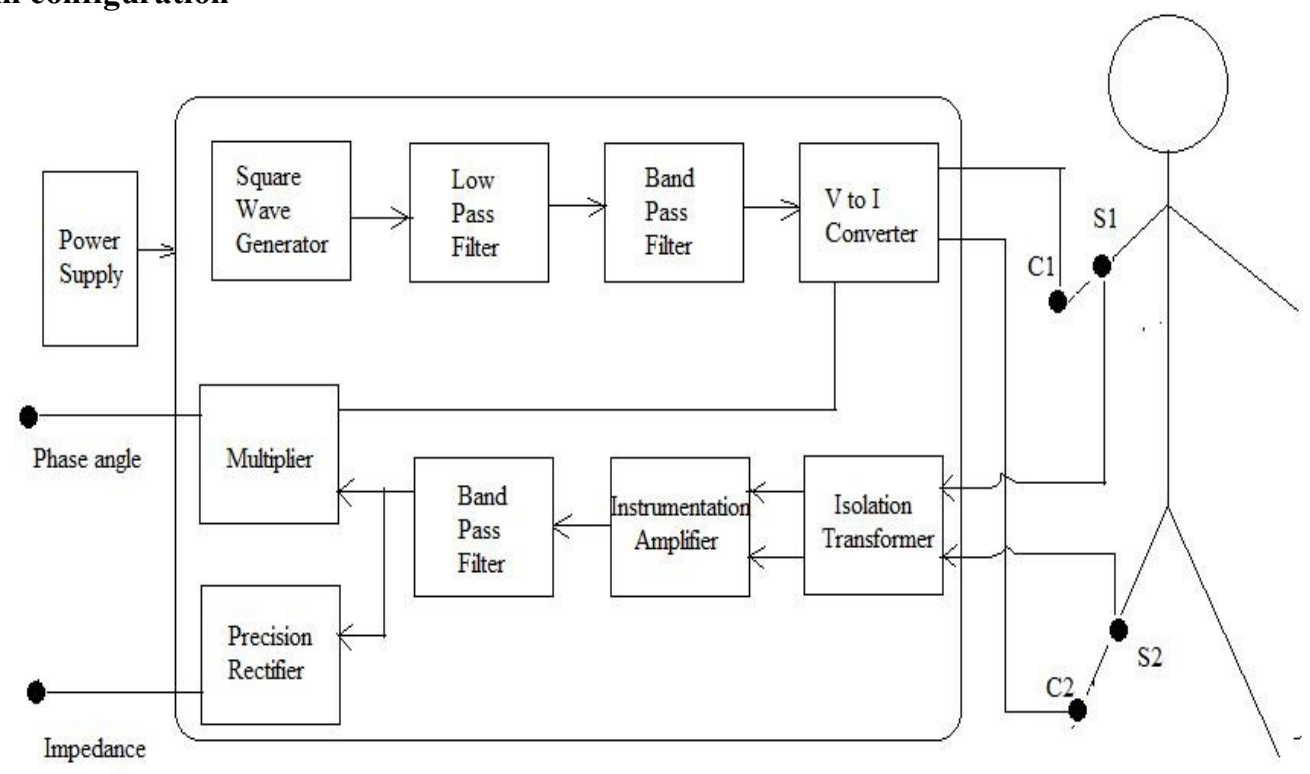

Fig. 3.1 Developed System Block Diagram

The Fig. 3.1 shows the developed system block diagram. The system is a battery operated portable device which uses DC battery for operation.

- Power supply: This block of the system consists of a rechargeable DC battery and a low voltage drop out regulator IC which supplies $+5 \mathrm{~V}$ DC supply. As the whole designed system requires $\pm 5 \mathrm{~V}$ supply, the $+5 \mathrm{~V}$ output of the regulator is given to DC to DC converter which supplies $\pm 5 \mathrm{~V}$. The converter also provides isolation. 
- Square Wave Generator: The square generator is a simple astable multivibrator operating at $+5 \mathrm{v}$ supply which generate a square wave of $46 \mathrm{KHz}$ which is required for the estimation of body impedance.

- Low Pass Filter: The low pass filter is designed using opamp to convert the square wave to sine wave with a cut of frequency of $49 \mathrm{KHz}$. The output of the low pass filter is a sawtooth wave. For smoothing further an opamp based band pass filter is implemented for generation of sine wave.

- Band Pass Filter: After converting the square wave into sawtooth wave for smoothing a band of frequencies between 40 to $60 \mathrm{KHz}$ is passed using opamp based band pass filter. Further current sine wave is generated by conversion using $\mathrm{V}$ to I converter.

- V to I converter: As a current is to be generated to pass through the human body the voltage sine wave is converted to current sine wave using opamp. The low pass filter, band pass filter and V to I converter is compiled using one quad opamp IC AD8044. This current is passed to the body through pair of current electrodes. The Electrodes used are band type electrodes made from copper mesh and coated with tin. As the current flows through the body using electrodes the voltage induced in the body is measured using pair of voltage electrodes which are fed to an isolation transformer.

- Isolation Transformer: It is a 1:1 ferrite core transformer which suppresses the $50 \mathrm{~Hz}$ frequency which provides isolation between human body and an electronic circuit.

- Instrumentation amplifier: The differential signals from the transformer are fed to the instrumentation amplifier which amplifies the signals from the body.

- Band pass filter: the amplified signal is suppressed from the unwanted frequencies using band pass filter.

- Precision Rectifier: To calculate the Impedance value from the signal i.e. to extract the amplitude information from the band pass filter output the rectifier removes out the negative cycle and converts the signal from AC to DC. This DC value extracted is further divided into half its value using a resistor network. As before the band pass filter, rectifier and the resistor networks are compiled in the second quad opamp IC AD8044. The output is further calculated for Impedance output.

- Multiplier: The output of the instrumentation amplifier and one of the differential output from the V to I converter is fed to the multiplier which calculates the phase angle between the input current and output induced voltage from the body. This phase angle is used to calculate the resistance and reactance component of the Bioimpedance analysis technique.

\subsection{PCB fabrication and component assembling}

Circuit design is prepared using software express PCB. From the circuit diagram PCB layout is made using same software. A one sided PCB is developed from the PCB layout achieved from the software. The PCB is of $130 \mathrm{~mm} \times 100 \mathrm{~mm}$ size. Further the components were assembled one by one on the PCB and were soldered. Further one by one each part of the circuitry is tested and calibrated for their appropriate output. After complete testing of the circuit the impedance and the phase angle for different female and male individuals are tabulated.

\section{Result}

This subsection includes the test results of the frequency generator, filters, amplifier, and rectifiers of the developed circuit. The square wave generator was designed for $50 \mathrm{kHz}, 5 \mathrm{~V}$. The output of the circuit is $45 \mathrm{kHz}, 2.8 \mathrm{v}$ this is due to resistors tolerance, and external noise interferences as shown in the Fig. 4.1. The output of the square wave generator is fed to a low pass filter which is designed for the $48 \mathrm{kHz}$ cut off frequency. The output of the low pass filter is $45 \mathrm{Khz}, 360 \mathrm{mV}$ as shown in the Fig. 4.2. The band pass filter is fed with the output of the low pass filter. The band pass filter is designed for the frequency 40 to $60 \mathrm{KHz}$. It is used to achieve exact shape of the sine wave as the output of the low pass filter for square wave input is exponential. The output of the band pass filter is tuned to 1Vpp shown in the Fig. 4.3. The output of the band pass filter is fed to a V to I converter to convert the voltage waveform to a current waveform. The Fig.4.4 and 4.5 shows the two current waves which are injected into the body using pair of current electrodes. The Current waveforms are $1 \mathrm{~mA}, 1 \mathrm{Vpp}$ in nature. The voltage induced in the body is picked up using pair of electrodes. The output signal is amplified using instrumentation amplifier which is designed for a gain of 100 . The output of the instrumentation amplifier is $8 \mathrm{~V}$ as shown in Fig. 4.6. The amplified signal is fed to the band pass filter and the rectifier for DC conversion the output of the band pass filter is shown in Fig. 4.7. This signal is rectified using a full wave rectifier for DC conversion. The output of the rectifier is the voltage induced in the body and impedance is calculated as explained in section 4.1. The output of the rectifier is as shown in figure 4.8. This value is measured and calculated for impedance. 


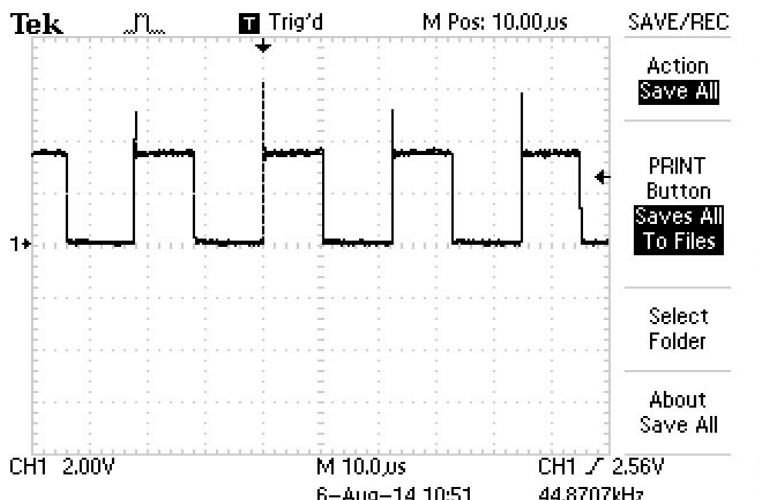

Fig. 4.1 Frequency generator output.

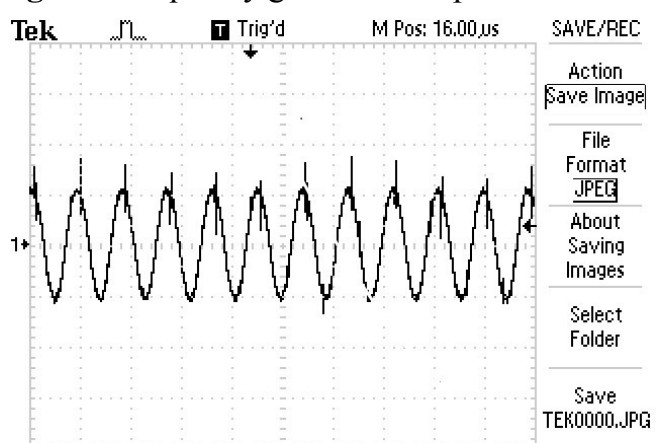
M 25.0us $\mathrm{CH} 1 / 176 \mathrm{~m}$ Current Folder is A: '

Fig. 4.3 Band pass filter output.
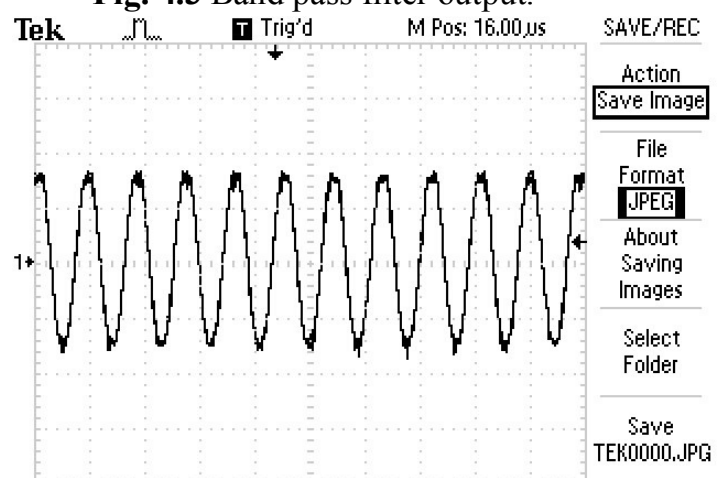
Current Folder is A:

Fig. 4.5 Input Current waveform 2.

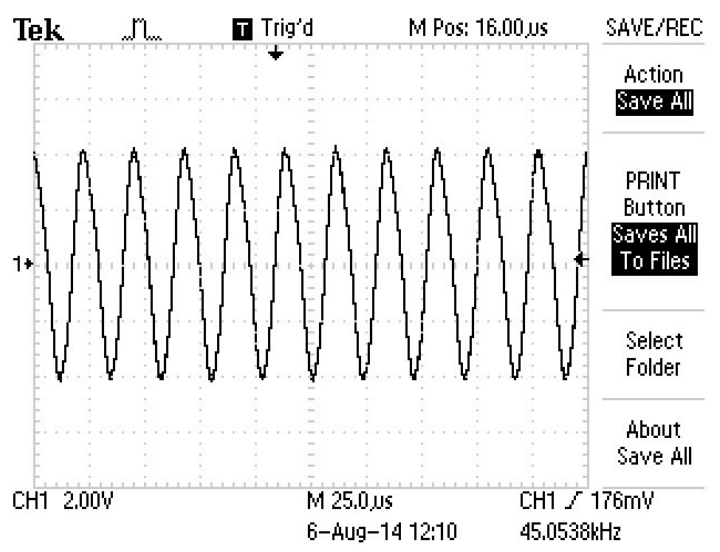

Fig. 4.7 Band pass filter 2 output.

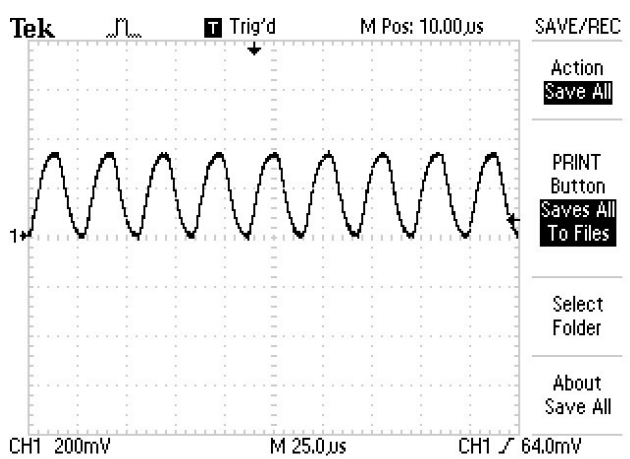

Fig. 4.2 Low pass filter output.

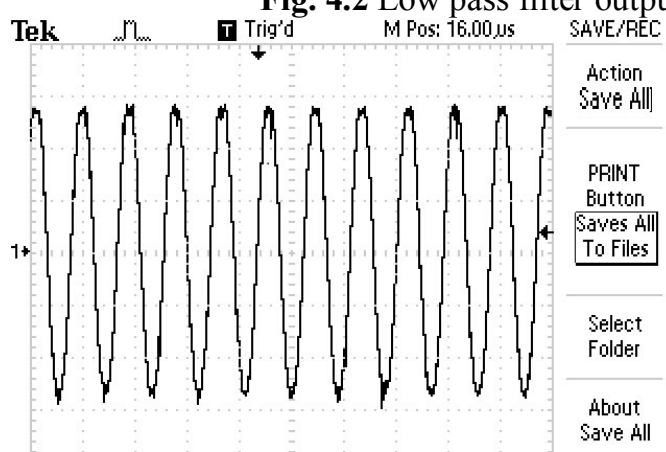

CH1 $500 \mathrm{ml}$

M 25.0us

$\mathrm{CH} 1 / 176 \mathrm{~m}$

Current Folder is A:

Fig. 4.4 Input Current waveform

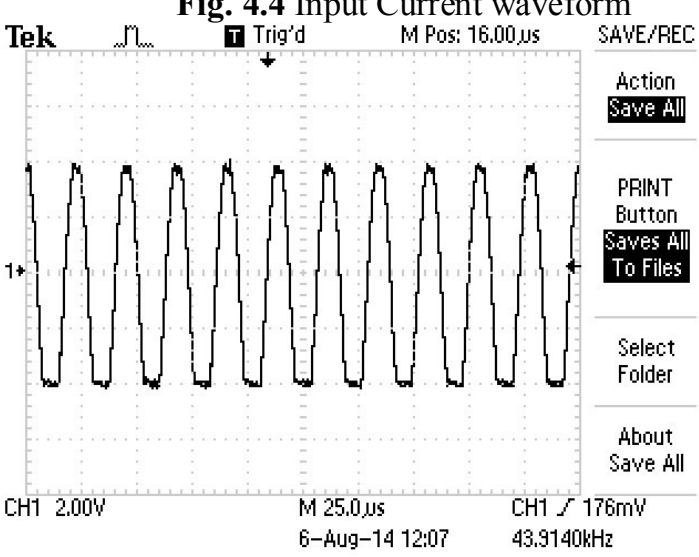

Fig. 4.6 Amplified Signal.

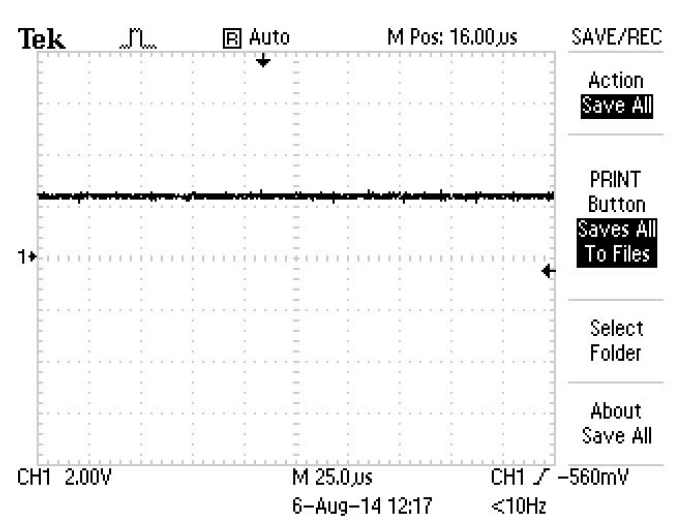

Fig. 4.8 Rectifier output. 


\subsection{Calculations}

The induced voltage in the body and the resistive component of the body impedance was measured from 60 healthy subjects in a lab-setup. The bio impedance was measured using equation (4.2). The bio impedance meter was calibrated by passing a current through series network of $100 \Omega, 1 \mathrm{k} \Omega$ and $100 \Omega$ resistors the induced voltage across $1 \mathrm{k} \Omega$ resistor was fed to the amplifier and consecutive circuits. The current calibration factor was calculated using (4.1) and was used for measurement of bio impedance. The bio impedance was measures using (4.2)

$$
\begin{aligned}
& \text { CalibrationFactor }=\frac{V d d_{(1 K \Omega)}}{1 K \Omega} \\
& Z=\frac{V d d}{\text { CalibrationFactor }}
\end{aligned}
$$

The phase angle between the injected current and the induced voltage was measured. The phase angle is calculated using equation (4.3). The calculation of the resistance value $\mathrm{R}$ is as explained in the previous chapter.

$$
\text { PhaseAngle }=\cos ^{-1} \frac{R}{Z}
$$

The developed instrument was used to measure the bio impedance from 60 healthy subjects including 25 males of 15-80 age group and 35 females of 12-80 age group. The results are tabulated below.

Table 4.1 Instrument results for female individuals

\begin{tabular}{cccccccc}
\hline $\begin{array}{c}\text { Sr } \\
\text { No }\end{array}$ & $\begin{array}{c}\text { Age } \\
\text { (Years) }\end{array}$ & $\begin{array}{c}\text { Weight } \\
(\mathbf{K g})\end{array}$ & $\begin{array}{c}\text { Height } \\
(\mathbf{c m})\end{array}$ & $\begin{array}{c}\text { Vdd } \\
\text { (v) }\end{array}$ & $\begin{array}{c}\mathbf{R} \\
(\mathbf{v})\end{array}$ & $\mathbf{Z}$ & $\begin{array}{c}\text { Phase } \\
\text { angle }\end{array}$ \\
\hline 1 & 12 & 30 & 121 & 1.92 & 0.56 & 0.97 & 54.73 \\
2 & 70 & 50 & 147 & 1.71 & 0.36 & 0.77 & 62.12 \\
3 & 78 & 51 & 147 & 1.25 & 0.25 & 0.41 & 52.42 \\
4 & 79 & 65 & 150 & 1.89 & 0.25 & 0.94 & 74.57 \\
5 & 80 & 52 & 150 & 1.78 & 0.48 & 0.84 & 55.15 \\
6 & 28 & 42 & 152 & 1.69 & 0.23 & 0.75 & 72.14 \\
7 & 62 & 75 & 152 & 1.81 & 0.4 & 0.87 & 62.62 \\
8 & 32 & 67 & 155 & 1.65 & 0.41 & 0.75 & 56.86 \\
9 & 78 & 40 & 155 & 1.85 & 0.26 & 0.9 & 73.2 \\
10 & 70 & 70 & 157 & 1.78 & 0.37 & 0.84 & 63.86 \\
\hline
\end{tabular}

Table 4.2 Instrument results for male individuals

\begin{tabular}{cccccccc}
\hline Sr No & $\begin{array}{c}\text { Age } \\
\text { (Years) }\end{array}$ & $\begin{array}{c}\text { Weight } \\
\text { (Kg) }\end{array}$ & $\begin{array}{c}\text { Height } \\
(\mathbf{c m})\end{array}$ & $\begin{array}{c}\text { Vdd } \\
\text { (v) }\end{array}$ & $\mathbf{R}$ & $\mathbf{Z}$ & $\begin{array}{c}\text { Phase } \\
\text { angle }\end{array}$ \\
\hline 1 & 28 & 72 & 170 & 1.52 & 0.36 & 0.6 & 53.13 \\
2 & 17 & 76 & 173 & 1.72 & 0.43 & 0.78 & 56.54 \\
3 & 47 & 67 & 173 & 1.65 & 0.42 & 0.72 & 54.31 \\
4 & 51 & 68 & 173 & 1.73 & 0.38 & 0.79 & 61.24 \\
5 & 62 & 68 & 173 & 1.66 & 0.36 & 0.73 & 38.49 \\
6 & 51 & 68 & 173 & 1.73 & 0.38 & 0.79 & 61.24 \\
7 & 62 & 68 & 173 & 1.66 & 0.36 & 0.73 & 38.49 \\
8 & 30 & 70 & 178 & 1.75 & 0.32 & 0.81 & 66.73 \\
9 & 79 & 93 & 178 & 1.45 & 0.29 & 0.55 & 58.17 \\
10 & 21 & 70 & 180 & 1.83 & 0.5 & 0.88 & 55.37 \\
\hline
\end{tabular}

\section{Conclusion}

In the course of this project an electronic circuitry which is required for the recording of impedance and phase angle is developed. The output waveforms of every stage of the developed circuit are included in the results. The practical results of various circuits are matching with $40 \%$ error. With the help of the developed system the impedance and phase angle values of different healthy individuals are recorded. These recorded results are tabulated.

\section{Acknowledgement}

I would like to take this opportunity to thank MGM's College of Engineering trust and Principal Dr. S K Narayankhedekar for giving me an opportunity to carry out my dissertation work. I wish to acknowledge my extreme gratitude to my guide Prof. U. R. Bagal for guiding me throughout the work. I have been greatly benefited by his valuable suggestion and ideas. I express my deep sense of gratitude towards Dr. G. D. Jindal for his valuable guidance and encouragement.

I also express my thanks to Prof. Aarti Bokade, Prof Bipin Mokal, Mr. Nazim momin, Mr. Suryapal, Mr. V.V. Gaikwad and my classmates for giving a helping hand and encouragement during the work. 


\section{References}

[1] Edwin K. Bioelectrical Impedance Anal. and Body Composition [Online]. Available: http://www.thebody.com

[2] Paul B P and Maria A, "Use of Bioelectrical impedance anal. in clinical manage. of malnutrition", Amer. J. of Clinical Nutrition, pp. 485-488

[3] Paul D, "Changes in fat free mass during weight loss determined by bio impedance Anal. and by densitometry," The Amer. J. of Clinical Nutrition, vol. 49, pp. 33-36, 2014

[4] Baker L. E, "Principles of the impedance technique", IEEE Eng. in Medicine and Biology Mag. , vol. 8, no. 1, pp. 11-15

[5] Schwan H. P. and Kay C. F, "The conductivity of living tissues", Annals of the New York Academy of Sci. , vol. 65, no. 6, pp. 1007-1013

[6] Baumgartner R. N et. al., "Bioelectric impedance phase angle and body composition", The Amer. J. of Clinical Nutrition, vol. 48, pp. 16-23

[7] Lukaski, H. C. and Bolonchuk W. W, "Theory and validation of the tetrapolar bioelectrical impedance method to assess human body composition", J. Appl. Physiol., vol. 60, no.4, pp.1327-1332, 1986

[8] Barnett A, "The basic factors in proposed electrical methods for measuring thyroid function, III. The phase angle and the impedance of the skin", Western J. of Surgery, Obstetrics, and Gynecology, vol. 45, pp. 540-554

[9] Spence J. A. et. al., "Changes during hemodialysis in total body water, cardiac output and chest fluid as detected by bioelectric impedance anal.", Amer. Soc. for Artificial Internal Organs, vol. 25, pp. 51-55

[10] Hoffer E. C. et. al., "Correlation of whole-body impedance with total body water volume", J. of Applied Physiology, vol. 27, pp. $531-534$

[11] Nyboer J, "Elect. Impedance Plethysmography”, Charles C Thomas, Springfield, IL

[12] Thomasset A, "Bio-electrical properties of tissue impedance measurements in clinical medicine", Lyon Medical, vol. 207, pp. 107118

[13] Ducrot H., et. al., "Determination of extracellular fluid volume in man by measurement of whole body impedance", Presse Medical, vol. 51, pp. 2269-2272

[14] Rudolph J. L, "Introduction to Bio Impedance Anal., " Principle and Fundamentals of Bioelectrical Impedance Anal. , USA: Boomix, ch.1, pp. 7-36

[15] Paul B P and Maria A, "Use of Bioelectrical impedance anal. in clinical manage. of malnutrition", Amer. J. of Clinical Nutrition, pp. $485-488$

[16] B R. Patil, "Bioelectrical Impedance Anal. for Bone Density Measurement," Ph.D. dissertation, Elect. Eng. , Mumbai University, Mumbai, Maharashtra, 2008 